\title{
Coronavirus disease 2019 (COVID-19) during pregnancy in patients with rheumatic diseases
}

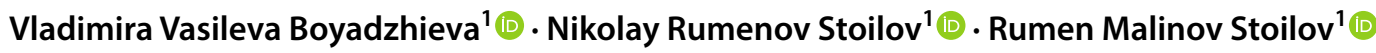

Received: 10 May 2020 / Accepted: 31 August 2020 / Published online: 15 September 2020

(c) Springer-Verlag GmbH Germany, part of Springer Nature 2020

\begin{abstract}
The novel coronavirus outbreak induces many concerns about the management of pregnancy, as well as rheumatic and musculoskeletal diseases. The very rapid spread of the infection throughout all inhabited continents leads to a fast-growing number of infected with SARS-CoV-2 and requires answers and special recommendations to the most vulnerable group of people: pregnant woman and patients on immunomodulatory or immunosuppressive treatment. A systematic literature search was performed in Embase, MEDLINE, and Scopus database for studies describing COVID-19 infection in pregnant women diagnosed with rheumatic and musculoskeletal diseases. From the 1,115 initially identified articles, we selected 29 publications in the English language, from which 18 were eligible according to the inclusion criteria. Limited number of cases and further researches are required to evaluate the risk of transmission of SARS-CoV-2 from mother to her infant as well as clinical features of infection in pregnant women. The conclusions of different authors, despite the small number of cases, suggest that there is no vertical transmission in women diagnosed with COVID-19 pneumonia. Although the World Health Organization recently reported that pregnant patients do not have a higher risk of infection than the rest of the population, Royal College of Obstetricians \& Gynecologists and The Royal College of Midwives for COVID-19 infection in pregnancy published Guidelines for pregnant women with suspected SARS-CoV-2 infection.

Considerations about patients with rheumatic diseases on the immunosuppressive treatment required European League Against Rheumatism, American College of Rheumatology, British Society for Rheumatology, and Australian Rheumatology Association to publish recommendations for patients with rheumatic diseases and COVID-19. These algorithms are very important to the medical society, but many concerns, absence of experience, and many questions are still unanswered and need time to be resolved and proceed successfully in this global pandemic situation.
\end{abstract}

Keywords COVID-19 $\cdot$ Pregnancy $\cdot$ Rheumatic diseases $\cdot$ Recommendations

\section{Introduction}

The global pandemic of the coronavirus disease 2019 (COVID-19) posed a challenge to many countries' health systems. In December 2019 a new public health threat, initially originated in Wuhan, China, was announced as severe

Vladimira Vasileva Boyadzhieva

Vladimira.boyadzhieva@gmail.com

Nikolay Rumenov Stoilov

dr_nstoilov@yahoo.com

Rumen Malinov Stoilov

rmstoilov@abv.bg

1 Clinic of Rheumatology, University Hospital "St. Ivan Rilski", Medical University of Sofia, 13 Urvich str, 1612 Sofia, Bulgaria acute respiratory syndrome coronavirus 2 (SARS-CoV-2), with clinical manifestation of severe viral pneumonia $[1,2]$. By the 11th of April, it had gone global and some 1610909 people in 212 countries had been registered with having the infection [3].

Due to the rapidly increasing number of cases and the very real risk of spread, the importance of this worldwide problem was quickly recognized and The Rheumatology Society decided to create an online platform called 'COVID-19 Global Rheumatology Alliance'-The global rheumatology community's response to the worldwide COVID-19 pandemic [4]. The registry was set up to be of help in the international effort to collect information pertinent to COVID-19 infection in patients with rheumatic diseases. The initiative is supported by the American College of Rheumatology (ACR), European League Against 
Rheumatism (EULAR), and more than 270 other societies, associations, foundations, and journals [4]. The recognition of the initiative demonstrates the social commitment and community support for patients with rheumatic disease and proven COVID-19 infection.

The site aims to collect information for all cases of COVID-19 in rheumatology patients, including those with mild or no symptoms. The clinicians report not only data for adult cases, but also pediatric patients with proven infection. By the 10th of April, 360 cases are reported to the official COVID-19 Global Rheumatology Alliance: 234 by Global/ University of California San Francisco Registry (UCSF) and 126 by European / EULAR Registry [5]. By the beginning of August 2020, this number had rapidly increased to 4,293 patients with RMD, diagnosed with COVID-19. No case has been reported yet of a pregnant patient with rheumatic disease infected with the new virus, or at least according to the data available to the authors of this review.

However, the number of reports for pregnant women registered with COVID-19 infection was increasing worldwide which provoked obstetricians, gynecologists, and specialists in infectious diseases to publish information for healthcare professionals as well as guidelines and algorithms for management [6-9]. The rapid spread of the COVID-19 pandemic in 212 countries has raised questions about the safety of pregnant women as well as many concerns about fetal health. The pregnant patients with rheumatic diseases have immunocompromised status, which in combination with the need for specific treatment with corticosteroids, non-steroidal anti-inflammatory drugs, conventional synthetic or biological disease-modifying anti-rheumatic drugs (DMARDS), could make them more susceptible to infection with COVID-19 than the general population. Despite the growing international experience and the increasing number of pregnant women diagnosed with SARS-CoV-2, little is still known about the impact of the novel coronavirus on pregnancy, especially in patients with rheumatic and musculoskeletal diseases (RMD) $[10,11]$.

One of the most important determinants of infectious risk, which leads to many complications during RMD is the presence of comorbidity $[12,13]$. Misra et al. summarized in "Rheumatologists' perspective on coronavirus disease 19 and potential therapeutic targets" that environmental factors and comorbidity presumably increase the severity of COVID-19 [13]. Moreover, cardiovascular manifestations, as well as diabetes mellitus, interstitial lung disease, chronic kidney diseases, and renal failure are a major risk factor for clinical features and severity in viral infection [13-15]. A recent study of Yao $\mathrm{H}$ et al. concludes that older males with chronic concomitant diseases were more likely to develop severe SARS-CoV-2 infection [16].

Recently, the World Health Organization (WHO and Centers for Disease Control and Prevention (CDC) have announced that pregnant women have the same risk as to the general population [17-19]. However, none of the statements is clear on whether pregnant patients with rheumatic diseases with or without comorbidities are at increased risk of infection with COVID-19, possibly due to the lack of sufficient data needed for analysis.

The purpose of this systematic review is to provide an overview of the current data for COVID-19 in patients with rheumatic diseases and pregnancy and answer the concerns of medical professionals and in particular rheumatologists in this difficult situation.

\section{Search strategy}

We searched in Embase, MEDLINE, and Scopus on 11. 04. 2020 for articles in English, describing COVID-19 infection in pregnant women diagnosed with rheumatic and musculoskeletal diseases without restriction to publication date. We used the following combination of keywords in the search strategy: "pregnancy", "novel coronavirus", "COVID-19", "SARS-CoV-2", "rheumatic diseases". The search was performed by two independent authors who identified 1,115 results, but most of them were excluded due to the study not including pregnancy, RMD, humans, or language other than English.

After screening the records and paying particular attention to the keywords, a total of 794 publications were excluded from the current systematic review. Our main focus was studies with inclusion criteria reporting cases of pregnant women with COVID-19, patients with rheumatic diseases, and recommendations for these groups of patients in the current pandemic situation.

Initially, we identified 29 publications in the English language: reviews of the literature, case reports, guidelines, original articles, and correspondence that were reviewed in full text. After the selection of the publications for eligibility, based on the aforementioned criteria, 11 articles were excluded (Fig. 1). We found the recommendations of the Royal College of Obstetricians \& Gynecologists (RCOG) and The Royal College of Midwives for COVID-19 infection in pregnancy, as well as Guidelines for pregnant women with suspected SARS-CoV-2 infection which is included in the summary of this review $[6,9,20]$.

\section{COVID-19 transmission and pregnancy}

Recently, numerous concerns were raised in regards to the health of pregnant women and the safety of their children and have come to the fore after the rapid spread of COVID-19 throughout the five continents. Despite there being no reported patients with rheumatic disease infected during pregnancy with COVID-19, there is still the real 
Fig. 1 Systematic review flowchart

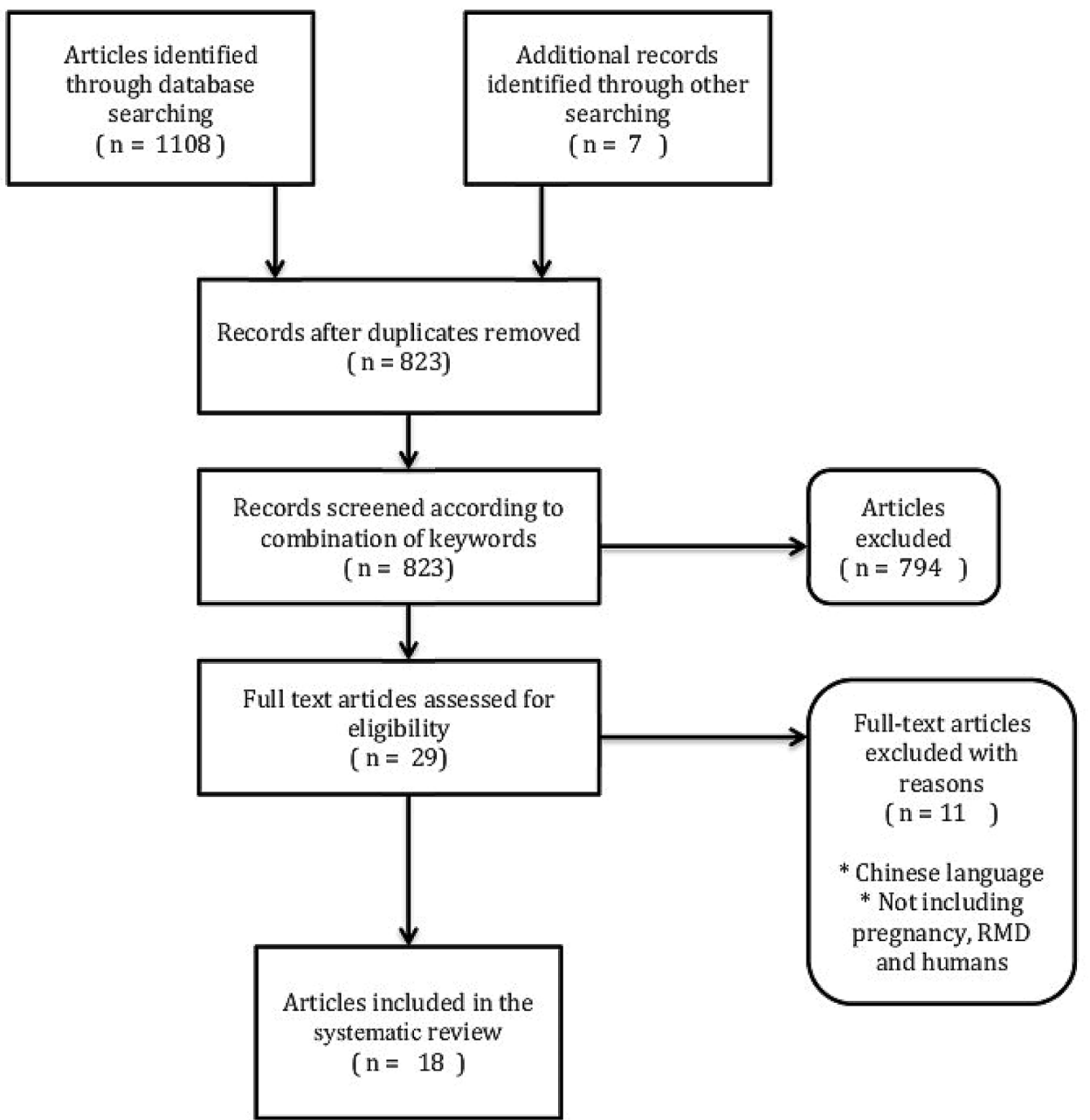

possibility of this happening as the numbers are increasing each day and the possibility of avoiding contamination seems impossible.

Since the beginning of 2020, 12 articles have been published presenting case reports, reviews, correspondences, and guidelines for the management of SARS-CoV-2 infection in pregnancy [6-11, 17, 21-25].

In most of the reported cases, we find evidence proving that the transmission of SARS-CoV-2 infection is from humans to humans $[6,22]$. Usually through direct close contact with infected persons' respiratory secretions which can enter the eyes, mouth, nose, and airways [6]. The distance required to avoid this contact is within $2 \mathrm{~m}$. However, recently published correspondence in The New England Journal of Medicine by Meselson announced that aerosols from infected persons can pose a risk of inhalation even over greater than this distance and especially indoors if there is poor ventilation [26]. The infectious risk increases with the higher quantity of the novel coronavirus entering the body. The SARS-CoV-2 can be isolated from respiratory secretions, feces, and fomites [6, 7].
The second route of spreading the disease is via touching contaminated surfaces, objects, or the hand of an infected person and subsequent transmission to one's eyes, mouth, or nose [6].

In pregnant women, one of the widely discussed topics is the vertical transmission of COVID-19 infection from mother to her child antenatally or intrapartum. Chen et al. report in a retrospective review of nine pregnant women with laboratory-confirmed COVID-19 pneumonia absence of evidence for vertical transmission. The amniotic fluid, cord blood, and neonatal throat swab sample were tested, as well as breastmilk samples after the first lactation - all of the samples were negative for the virus. All nine patients gave birth via caesarean section in their third trimester [11]. The conclusion of the author, despite this small number of cases, suggests that there is no vertical transmission in women diagnosed with COVID-19 pneumonia [11].

$\mathrm{Nan} \mathrm{Yu}$ and colleagues report In The Lancet Infectious Diseases, clinical features of seven pregnancies and obstetric and neonatal outcomes in these patients infected with SARSCoV-2 in Wuhan [27]. All of the cases had similar symptoms 
to those of the other adults infected with COVID-19 and were treated with antiviral therapy, oxygen supplementation, and isolation. All of the babies were delivered by caesarean sections without complications, and the children were born in good condition [27]. Three of the newborns were tested for SARS-CoV-2 and one was positive for the infection $36 \mathrm{~h}$ after birth [27]. These findings are a prerequisite for hypothetical vertical transmission, but insufficient data at this stage does not allow general conclusions.

Another descriptive study by Zhu and colleagues, report 10 neonates (including two twins) born by 9 pregnant women with COVID-19 [28]. Seven of the babies were delivered by cesarean section and three by vaginal delivery (one pair of twins and one single baby) [28]. Pharyngeal swab specimens were collected from nine of the neonates and all of them were negative, including those from babies who were delivered "per vias naturales" [28]. Concerns about the opportunity of higher infectious risk of COVID19 vertical transmission through vaginal delivery are still reasonable due to different scientific reports (Table 1). However, most of them describe negative results for SARS$\mathrm{CoV}-2$ in neonates after the delivery.

Breslin et al. confirm the absence of vertical transmission in 18 women who have delivered including 4 symptomatic patients for COVID-19 and 14 initially asymptomatic. Fifteen neonates were negative for SARS-CoV-2, tested on the same day of birth and two infants had unclear results on the day of life 0 , and subsequently tested and negative on day
1-2. "Indeterminant" test result was found in one baby, who was clinically assessed as "healthy" and accepted for "presumptive negative" [29]. None of the neonates had positive antibodies for IgM or IgG COVID-19 [29].

In contrast to these results, two studies have published evidence of positive IgM SARS-CoV-2 in the serum of newborn at birth (Table 1) [30, 31]. IgM does not cross the placenta, so it is very likely to be related to the neonatal immune response to in utero infection [6].

The discussion of whether vaginal delivery increases the infectious risk exists before the current COVID-19 pandemic. Since 2005, Yudin and colleagues described a 33-year old pregnant woman diagnosed with SARS at 31 weeks of gestation and clinical symptoms of fever, a dry cough, and patchy infiltrates on chest X-ray. No further complications were observed and no oxygen supplementation was needed. A healthy female baby was delivered vaginally with no evidence of infection [32].

All of the described reports and conclusions are based on a small number of cases and further researches is required to evaluate the infectious risk and algorithms to proceed in this global pandemic situation.

\section{Clinical features of COVID-19 in a pregnant woman}

According to the Guidance for healthcare professionals on coronavirus (COVID-19) infection in pregnancy, published by the RCOG and Royal College of Midwives, pregnant

Table 1 Summary of reported cases, possible vertical transmission and patient's treatment

\begin{tabular}{|c|c|c|c|c|c|c|}
\hline & $\begin{array}{l}\text { Chen } \mathrm{H} \text { et al. } \\
(2020)^{11}\end{array}$ & $\begin{array}{l}\text { Nan Yu et al. } \\
(2020)^{27}\end{array}$ & $\begin{array}{l}\text { Zhu H et al. } \\
(2020)^{28}\end{array}$ & $\begin{array}{l}\text { Breslin N } \\
\text { et al. (2020) } \\
29\end{array}$ & $\begin{array}{l}\text { Dong L et al. } \\
(2020)^{30}\end{array}$ & $\begin{array}{l}\text { Zeng } \\
\text { H et al. } \\
(2020)^{31}\end{array}$ \\
\hline Number of infected women with COVID-19 & $9(100 \%)$ & $7(100 \%)$ & 9 & 18 & 1 & 6 \\
\hline Number of born neonates & $9(100 \%)$ & $7(100 \%)$ & 10 & 18 & 1 & 6 \\
\hline Cesarean section delivery & $9(100 \%)$ & $7(100 \%)$ & 7 & 9 & 1 & 6 \\
\hline Vaginal Delivery & 0 & 0 & 3 & 9 & NA & NA \\
\hline Number of tested neonates for COVID-19 PCR & 9 & 3 & 10 & 18 & 1 & 6 \\
\hline Nasopharyngeal swab positive for COVID-19 neonates & 0 & 1 & 0 & 0 & 0 & 0 \\
\hline COVID-19 IgG positive neonates & 0 & NA & NA & 0 & 1 & 5 \\
\hline COVID-19 IgM positive neonates & 0 & NA & NA & 0 & 1 & 2 \\
\hline Breastmilk samples positive for COVID -19 & 0 & NA & NA & NA & NA & NA \\
\hline Vertical transmission & No & Yes & No & No & Yes & Yes \\
\hline Oxygen support & $9(100 \%)$ & $7(100 \%)$ & - & $1(2 \%)$ & $1(100 \%)$ & - \\
\hline Antiviral therapy & $6(67 \%)$ & $7(100 \%)$ & $5(56 \%)$ & - & $1(100 \%)$ & - \\
\hline Antibiotic therapy & $9(100 \%)$ & $7(100 \%)$ & - & $2(5 \%)$ & $1(100 \%)$ & - \\
\hline Hydroxychloroquine & - & - & - & $2(5 \%)$ & - & - \\
\hline Corticosteroid treatment & 0 & $5(71 \%)$ & - & - & $1(100 \%)$ & - \\
\hline Interferon & 0 & $7(100 \%)$ & $1(11 \%)$ & - & - & - \\
\hline Chinese medicine & 0 & $7(100 \%)$ & - & - & - & - \\
\hline
\end{tabular}

*PCR polymerase chain reaction 
women do not appear to be more susceptible to coronavirus infection than the rest of the population [6]. However, the immune's system response to viral infection during pregnancy makes these women vulnerable to COVID-19 and can cause more severe symptoms [6,33].

Most of the pregnant women experience flu-like symptoms with a cough, shortness of breath, fever, anosmia, headache, and diarrhea [6, 23, 27]. In some cases, more severe complications such as pneumonia and marked hypoxia can occur.

Breslin and colleagues presented a series of 43 confirmed cases of pregnant women with COVID-19 admitted in New York City hospitals who showed a similar pattern of disease severity compared to the general population: 37 women (86\%) had mild disease symptoms, four (9.3\%) experienced severe disease symptoms and two (4.7\%) was in critical condition [29]. In comparison to the rest of the adult population described by $\mathrm{Wu}$ et al. these values are similar: $80 \%$ had mild, $15 \%$ severe and 5\% critical presentation of the disease [34]. Chen L. et al. confirms similar data to the data presented by $\mathrm{Wu}$ and colleagues, according to which 109 (92\%) of pregnant cases had mild disease, $9(8 \%)$ had severe symptoms and one patient received noninvasive mechanical ventilation due to critical course of the disease [35]. Most of the publications to date report predominantly mild infection with COVID-19 in pregnant women; however, there have been case reports for severe infection requiring ventilation and extracorporeal membrane oxygenation [36].

According to clinical features of COVID-19 infection in pregnant women, most of the studies reported good outcomes, but all of the enrolled patients were in the third trimester of the pregnancy and had predominantly mild symptoms [37]. Although Chen L. et al. described in the study population of 118 women, there were 3 spontaneous abortions, 2 ectopic pregnancies and 4 induced abortions (all due to the concerns of SARS-CoV-2 infection), a total of 68 patients delivered during the study period and had 70 births [35].

The most recent report of the Intensive Care National Audit and Research Centre (ICNARC) in the United Kingdom (UK) described 5578 patients who were admitted to critical care settings, diagnosed with COVID-19. From them, currently pregnant were 16 and recently pregnant (within 6 weeks) were 21 [38]. Despite the described by ICNARC pregnant woman in critical condition, Liu D and colleagues reported cases of COVID-19 pneumonia in pregnancy which are milder and consequent good recovery [39]. There are currently no reported deaths in pregnant women due to COVID-19 infection.

Historically, in a previous 2002-2003 coronavirus pandemic, Wong et al. describe pregnancy and perinatal outcomes in twelve women infected with SARS-CoV [40]. More than $50 \%$ of them reported abortion within the first trimester of the infection. In $40 \%$ of the pregnant patients in the second trimester was observed intrauterine growth restriction, and in $80 \%$ - preterm delivery. Furthermore, 25\% (3 women) died during the pregnancy [40]. In 2019, Alfaraj et al. described 11 cases of pregnant patients diagnosed with the Middle East respiratory syndrome (MERS-CoV). Perinatal death was reported in three of the women and $91 \%$ had adverse outcomes [24, 41]. Additional information about placental pathology was published in an article in Chinese by $\mathrm{Chen} \mathrm{S}$. et al. The authors reported 3 cases of women with COVID-19 who delivered by cesarean section [42]. The authors reported various fibrin deposition inside and around the villi with local syncytial nodule increases in all of the women. In one of the cases placental infarctions were found and in the other chorionic hemangioma was present like concomitant morphology [42]. All of the samples from the three placentas were tested for the nucleic acid of COVID-19 and the results appeared negative [42]. No clinical evidence of villitis or chorioamnionitis was observed in these cases [42].

A limited number of authors report cases of MERS and SARS-CoV infection during pregnancy. Most of them revealed an increased risk during the last trimester. Mullins et al. reported there was an increased risk of preterm birth indicated for maternal medical reasons after 28 weeks' gestation [43]. The authors conclude that preterm delivery was carried out in $47 \%$ of hospitalized pregnant women that were infected with COVID-19 [43]. Although further clarification and analyses are needed as to whether this was iatrogenic or spontaneous. In cases of iatrogenic delivery, it was predominantly for maternal indications related to the viral infection, but in some cases, there was evidence of fetal compromise and prelabour, preterm rupture of the membranes $[6,11,12]$.

Concerns about the possibility of hypercoagulation during pregnancy are increasing because of data from hospitalized patients with proven SARS-CoV-2 infection, which indicates changes in coagulation and a tendency to hypercoagulation [44]. Women during pregnancy after infection with SARS-CoV-2 likely have a higher risk of venous thromboembolism(VT) [45]. Other factors like smoking, movement limitations in consequence of isolation at home also increase the risk of thromboembolism. In pregnant patients with connective tissue diseases (like systemic lupus erythematosus (SLE) and /or antiphospholipid syndrome) the risk of hypercoagulation is even higher [46]. Therefore, concerns for this group of patients about possible maternal venous thromboembolism and additional infection with COVID-19 necessitate a combined approach and observation by a multidisciplinary team of specialists, as well as the prevention of hypercoagulation. The administration of low molecular weight heparin is the recommended option for the prevention of VT $[6,46]$. No case of a pregnant woman with a systemic connective tissue disease and infection with SARS-CoV-2 has been registered to date, which suggests 
that algorithms for treatment of this group of patients are yet to be developed.

\section{Recommendations for pregnant women diagnosed with COVID-19}

Ever since the novel coronavirus pandemic spread in over 212 countries and many authors reported an increasing number of pregnant women and neonates infected with SARS$\mathrm{CoV}-2$, the evidence showed good outcomes in most of the cases. However, the concerns about them necessitated the development of recommendations and guidelines for the prevention and treatment of suspected SARS-CoV-2 infected pregnant patients. Favre et al. proposed a detailed algorithm for health care providers [10].

In summary, these recommendations are divided into two parts: guidance for management of pregnant women with suspected COVID-19 and with confirmed SARS-CoV2 infection. The suggested algorithm in cases with suspected viral infection includes two main points. Firstly, all pregnant women, even asymptomatic, who have travelled abroad or had close contact with a patient with confirmed viral infection should be tested for the nucleic acid amplification test for SARS-CoV-2 [10]. Secondly, pregnant patients who are asymptomatic, but have confirmed infection, should be monitored at home for clinical symptoms for at least 14 days [10].

The recommendations for management in cases with confirmed SARS-CoV-2 infection suggest that asymptomatic and pregnant patients recovering from mild illness should be monitored twice a month. Ultrasound and Doppler assessments should be performed because of the potential risk for intrauterine growth restriction [10]. Furthermore, in case of complications like COVID-19 pneumonia, the patients should be treated at a tertiary care center by a multidisciplinary team. When quick organ failure caused by sepsis is demonstrated, patients should be transferred to an intensive care unit [10]. In the case of fetal distress, prompt caesarean delivery or termination (if it is legal before fetal viability) should be performed. An individual approach to the choice of delivery timing should be applied to each pregnant woman according to the gestational week and the condition of the mother and the fetus [10]. The authors recommend vaginal delivery via induction of labor, whenever is possible, to avoid maternal exhaustion and surgical complications in an already sick patient. Isolation for the neonates from their mothers who are positive for SARS-CoV-2 for at least 14 days or until viral shedding clears is recommended [10].

Further guidance for health care professionals, midwifery, and obstetric services caring for pregnant women was published by the Royal College of Obstetricians \& Gynecologists and The Royal College of Midwives [6]. The recommendations were made as a measure against disease.
They are divided into sections: general recommendations, advice-regarding social distancing and self-isolation, and recommendations regarding appointments and urgent visits to clinics and hospitals. The conclusion advises that all midwifery and obstetric services caring for a pregnant woman to maintain stringent social distancing [6]. Although visiting maternity services may cause significant anxiety about the possibility of infection with COVID-19, it is recommended that pregnant women do attend antenatal and postnatal care centers. Advice for many aspects of everyday life including maternal mental well-being, smoking cessation, and sources for self-help for anxiety and stress were published to support and organize pregnancy periods in the COVID-19 pandemic situation.

Breastfeeding is one of the topics that soon began to be discussed. The main risk in breastfeeding is the close contact between the mother and the child and the high potential of transmission of COVID-19. The RCOG recommends the infectious risk to be discussed with the parents and precautions should be taken to limit the viral spread to the baby [6]. Guidance and precautions for Infants' feeding include mothers' personal hygiene like washing her hands before touching the baby, breast pumps, or bottles. Secondly, the mother should avoid coughing or sneezing on the baby while feeding and wear a fluid-resistant surgical facemask while caring for the baby. Whenever she is using a breast pump, it is necessary to follow recommendations for pump cleaning after each use. If the mother feels unwell, she should ask someone who is not sick to feed the baby. For babies who are bottle-fed with formula or expressed milk, strict adherence to sterilization guidelines is recommended [6].

\section{Recommendations for patients with rheumatic diseases and COVID-19}

Over the last five months, since novel coronavirus spread all over the world, patients with rheumatic diseases face many challenges. The uncertainty and absence of information, as well as the characteristic of highly contagious viral illness with a mortality rate of $2 \%$, contributed to the increasing concern of patients with RMD about the use of immunomodulatory or immunosuppressive treatment.

During this time, 360 patients with RMD infected with SARS-CoV-2 were reported to the COVID-19 Global Rheumatology Alliance Registry of 7157 that were tested. This number includes not only sick patients but also asymptomatic who were positive for SARS-CoV-2. By the beginning of august 2020, the number of patients with RMD infected with SARS-CoV-2 had increased to 4293 (1783 by Global/(UCSF) and 2510 by European / EULAR Registry). Thirty-nine percent (91) of patients were hospitalized for SARS-CoV-2 infection and 7.63\% (10) died from complications [5]. The data from The COVID-19 
Global Rheumatology Alliance Registry reported detailed information from Global / UCSF registry (1783 cases): $39 \%$ (694) from all patients are diagnosed with Rheumatoid arthritis (RA), 9\% (165) with Psoriatic arthritis (PSA), 17\% (306) have SLE, 6\% (108) with Spondyloarthritis, 7\% (125) have vasculitis and 4\% (70) have Sjogren's syndrome [5]. Most of the patients are female $-1331(75 \%)$ and 451 $(25 \%)$ are males [5].

No reports are available for a pregnant patient diagnosed with rheumatic disease and infection with SASRS-CoV-2 to date [5].

According to the treatment regimen prior COVID-19 diagnosis-63\% (1120) were on conventional synthetic disease-modifying anti-rheumatic drugs (csDMARDS), $26 \%$ (471) on hydroxychloroquine, 31\% (547) administered biologic (bDMARDS), 5\% (87) Janus kinase inhibitors (JAK), $22 \%$ (394) took non-steroidal anti-inflammatory drugs (NSAIDs) and 33\% (594) on glucocorticoids [5]. The risk of infection reported by most of the randomized clinical trials is higher in patients on bDMARDS than these on csDMARDS [47, 48]. However, The COVID-19 Global Rheumatology Alliance Registry described more cases in patients with RMD infected with novel coronavirus while on treatment with csDMARDS. A likely explanation for this is that more patients are treated with conventional synthetic DMARDs in the patient population than with biological agents.

Considerations about the immunosuppressive treatment required EULAR, ACR, British Society for Rheumatology (BSR), and Australian Rheumatology Association (ARA) to publish recommendations for patients with rheumatic diseases and COVID-19 [49-52]. The major points include keeping strict personal hygiene (wearing a mask, washing hands regularly, avoid touching face), and social distancing (avoidance of handshakes and hugs). Moreover, patients with RMD should avoid public transport and large groups of people. In case symptoms such as fever or persistent cough appear, the patients should get tested for COVID-19. A routine rheumatology appointment can be postponed or can be performed by telephone (if it is not essential). According to the treatment, patients should avoid discontinuation of glucocorticoids, even in case of active infection. In the case of administration of Immune suppressive drugs like csDMARDS, bDMARDS, JAK inhibitors, glucocorticoids should not be stopped or the dose reduced except in case of infection [49-52].

In addition to the EULAR recommendations, The WHO recently published a paper on preventive measures for people in an area affected by an outbreak. Aide-memoire for infection prevention and control in a healthcare facility recommends personal hygiene, personal protective equipment, isolation precautions, aseptic techniques, cleaning and disinfection, sterilization and waste management, and antibiotic use protocol [53].
Although these recommendations are very important to rheumatologic society, many concerns, the absence of experience, and many questions are still unanswered. On the other hand, a growing number of articles and information about the pathogenesis of the viral infection as well as preliminary results from clinical trials with medications used for the treatment of rheumatoid arthritis give light and a whole new perspective to the current pandemic situation [14]. Chloroquine and hydroxychloroquine have been included in Chinese and French protocols for treating the complications of SARS-CoV-2 infection $[54,55]$. The Lancet Infectious Disease published on the third of April a correspondence regarding the potential use of Baricitnib for treatment of COVID-19 [56]. Moreover, a pilot study on the safety and clinical impact of Baricitinib therapy of COVID19 concludes that the use of this specific JAK inhibitor may limit the cytokine "storm" associated with the viral infection and may be useful for reduction of severity progression [57, 58]. Despite the promising results, the number of treated patients is still small and numerous questions about safety and clinical impact require careful consideration. Also, the potential role of Tocilizumab (anti-interleukin-6) for managing the cytokine storm, associated with the complications of infection with SARS-CoV-2, is still widely discussed [59]. A randomized clinical trial in phase III with anakinra demonstrated a significant survival benefit in patients with sepsis and suggested the potential use of anti-interleukin-1 in hyperinflammatory conditions and potential treatment for COVID-19 pneumonia [60]. Despite these promising data, there are still insufficient results to summarize general recommendations apply to most of the cases with RMD and COVID-19. One of the limitations of this review is the lack of reported cases of pregnant patients with rheumatic diseases. Another limitation is the lack of sufficient information and consensus on the treatment of patients with RMD and SARS-COV-2 infection. However, this review summarizes in detail the information from recent studies and purposefully informs about the initiatives of the rheumatological communities in the fight against COVID-19. Combining the recommendations of The Royal College of Obstetricians \& Gynecologists with those of EULAR, ACR, BSR, and ARA to give a more in-depth overview and a new perspective for the rheumatologist who decides how to treat his patients in this epidemic situation. Furthermore, a limited number of studies provide information on the various rheumatic diseases, the presence of comorbidity, and the consequences of SARS-COV-2 infection.

It is known, that PSA patients have higher cardiovascular risk than the general population [61, 62], so it is very likely that in combination with comorbidities like arterial hypertension and diabetes put this group of patients at higher risk of complications when infected with SARS-CoV-2 [63]. Moreover, psoriatic arthritis (PSA) is 
considered for an independent cardiovascular risk factor [64]. National Psoriasis Foundation (NPF) Medical Board COVID-19 recommendations for patients with psoriatic disease follow the global tendency and adhere to advice published by EULAR and ACR [63]. At this time, the NPF Medical Board does not recommend to patients with PSA to stop biologic therapy. Every case should be discussed with a rheumatologist and take an individual decision [63].

In contrast to algorithms recently published for inflammatory joint diseases, just a few recommendations are available for patients with SLE. Mathian et al. recently published in Annals of the Rheumatic disease observations based on the clinical course of COVID-19 in a series of 17 patients with SLE treated with hydroxychloroquine (HCQ) [65]. The authors conclude that therapy with HCQ does not seem to prevent COVID-19 and its complications in patients with SLE [65]. They can suffer from comorbidity like chronic kidney disease and obesity and consequently have more severe forms of COVID-19 [66, 67]. Recently, the ACR advise patients with SLE to talk to their rheumatologist and follow recommendations before the interruption of the therapy during SARS-CoV-2 infection [50, 68] However, there are no current specific advice and therapeutic strategy for patients with SLE.

\section{Conclusion}

Many concerns and questions are being continually addressed in an attempt to bring clarity and answers. Professional organizations have developed algorithms and recommendations to prevent morbidity and avoid complications in immunocompromised patients. Despite the amount of accumulated knowledge that has been collated in regards to infectious diseases, autoimmune answer, and different therapeutic strategies, which occur daily in clinical practice, the results from ongoing research are still being anticipated, as they would supplement the existing algorithms.

\begin{abstract}
Author contributions Two authors (VB) and (NS) performed the search and reviewed all articles in full text independently for relevance and consistency in accordance with the specified criteria. (VB) and (NS) wrote the manuscript and (RS) critically revised it. (VB), (NS) and (RS) approved the final version of the manuscript. No part of the manuscript including the table and figure were published elsewhere.
\end{abstract}

\section{Compliance with ethical standards}

No part of the manuscript including the table and figure were published elsewhere.

Conflict of interest The authors declare non-conflict of interest.

\section{References}

1. Li Q, Guan X, Wu P et al (2020) Early transmission dynamics in Wuhan, China, of novel coronavirus-infected pneumonia. $\mathrm{N}$ Engl J Med 382:1199-1207. https://doi.org/10.1056/NEJMo a2001316

2. Huang C, Wang Y, Li X et al (2020) Clinical features of patients infected with 2019 novel coronavirus in Wuhan China. Lancet 395:497-506

3. WHO. Coronavirus disease (COVID-19) situation report 82 https://www.who.int/docs/default-source/coronaviruse/situa tion-reports/20200411-sitrep-82-covid-19.pdf?sfvrsn=74a5d $15 \_2$

4. The COVID-19 Global Rheumatology Alliance. https://rheum -covid.org

5. The COVID-19 Global Rheumatology Alliance Global Registry. https://mailchi.mp/a6dc80671481/just-launched-the-covid-19global-rheumatology-registry-4810762

6. Coronavirus (COVID-19) infection and pregnancy. Version 7 (2020) Guidance for healthcare professionals on coronavirus (COVID-19) infection in pregnancy, published by the RCOG, Royal College of Midwives, Royal College of Paediatrics and Child Health, Public Health England and Health Protection Scotland. https://www.rcog.org.uk/globalassets/documents/ guidelines/2020-04-09-coronavirus-covid-19-infection-in-pregn ancy.pdf Accessed: 9 Apr 2020

7. Zhang W, Du R-H, Li B et al (2020) (2020) Molecular and serological investigation of 2019-nCoV infected patients: implication of multiple shedding routes. Emerg Microbes Infect 9:386-389. https://doi.org/10.1080/22221751.2020.1729071

8. Yongwen L, Kai Y (2020) Management of pregnant women infected with COVID-19. Lancet Infect Dis. https://doi. org/10.1016/S1473-3099(20)30191-2

9. Martinez-Portilla RJ, Gonce A, Hawkins - Villarreal A, Figueras F (2020) A Spanish - translated clinical algorithm for management of suspected SARS-CoV-2 infection in pregnant women. Lancet Infect Dis. https://doi.org/10.1016/S1473 -3099(20)30285-1

10. Favre G, Pomar L, Qi X, Nielsen-Saines K, Musso D, Baud D (2020) Guidelines for pregnant women with suspected SARSCoV-2 infection. Lancet Infect Dis. https://doi.org/10.1016/ S1473-3099(20)30157-2

11. Chen H, Guo J, Wang C et al (2020) Clinical characteristics and intrauterine vertical transmission potential of COVID-19 infection in nine pregnant women: a retrospective review of medical records. The Lancet 395:809-815. https://doi.org/10.1016/ S0140-6736(20)30360-3

12. Di Mascio D, Khalil A, Saccone G et al (2020) Outcome of Coronavirus spectrum infections (SARS, MERS, COVID) during pregnancy: a systematic review and meta-analysis. Am J Obstet Gynecol MFM. https://doi.org/10.1016/j.ajogmf.2020.100107

13. Misra DP, Agarwal V, Gasparyan AY, Zimba O (2020) Rheumatologists' perspective on coronavirus disease 19 (COVID19) and potential therapeutic targets. Clin Rheumatol 39:20552062. https://doi.org/10.1007/s10067-020-05073-9

14. Favalli EG, Ingegnoli F, De Lucia O et al (2020) COVID-19 infection and rheumatoid arthritis: Faraway, so close! Autoimmun Rev 19(5):102523. https://doi.org/10.1016/j.autrev.2020.102523

15. Onuora S (2020) New data emerging on outcomes for patients with COVID-19 and rheumatic diseases. Nat Rev Rheumatol 16:407. https://doi.org/10.1038/s41584-020-0463-8

16. Huang $\mathrm{Y}$ et al (2020) Clinical characteristics of 17 patients with COVID-19 and systemic autoimmune diseases: a retrospective study. Ann Rheum Dis. https://doi.org/10.1136/annrheumdi s-2020-217425 
17. Yang H, Wang C, Poon LC (2020) Novel coronavirus infection and pregnancy. Ultrasound Obstet Gynecol 55:435-437. https:// doi.org/10.1002/uog.22006

18. World Health Organization. Q\&A on coronaviruses (COVID19). https://www.who.int/news-room/q-a-detail/q-a-coronaviru ses. Accessed: 17 Apr 2020

19. Centers for Disease Control and Prevention. Coronavirus disease 2019 (COVID-19). Pregnancy and Breastfeeding. https://www. cdc.gov/coronavirus/2019-ncov/need-extra-precautions/pregn ancy-breastfeeding.html Accessed: 24 Apr 2020

20. Gasparyan AY, Ayvazyan L, Blackmore H, Kitas GD (2011) Writing a narrative biomedical review: considerations for authors, peer reviewers, and editors. Rheumatol Int 31(11):1409-1417. https:// doi.org/10.1007/s00296-011-1999-3

21. Wang X, Zhou Z, Zhang J, Zhu F, Tang Y, Shen X (2020) A case of 2019 Novel Coronavirus in a pregnant woman with preterm delivery. Clin Infect Dis. https://doi.org/10.1093/cid/ciaa200

22. Li Y, Zhao R, Zheng S et al (2020) Lack of vertical transmission of severe acute respiratory syndrome coronavirus 2 China. Emerg Infect Dis. https://doi.org/10.3201/eid2606.200287

23. Saccone G, Carbone F, Zullo F (2020) The novel coronavirus (2019-nCoV) in pregnancy: what we need to know. Eur J Obstetr Gynecol Reprod Biol. https://doi.org/10.1016/j.ejogr b.2020.04.006

24. Liu D, Li L, Wu X et al. (2020). Pregnancy and Perinatal Outcomes of Women with COVID-19 Pneumonia: A Preliminary Analysis. Available at SSRN: https://ssrn.com/abstract=35487 58. Accessed: 29 Feb 2020

25. Sutton D, Fuchs K, D’Alton M, Goffman D (2020) Universal screening for SARS-CoV-2 in Women admitted for delivery. $\mathrm{N}$ Engl J Med. https://doi.org/10.1056/NEJMc2009316

26. Meselson M (2020) Droplets and aerosols in the transmission of SARS-CoV-2. N Engl J Med. https://doi.org/10.1056/NEJMc 2009316

27. Nan Yu, Li Wei, Qingling K et al (2020) Clinical features and obstetric and neonatal outcomes of pregnant patients with COVID-19 in Wuhan, China: a retrospective, single-center, descriptive study. Lancet Infect Dis. https://doi.org/10.1016/ S1473-3099(20)30176-6

28. Zhu H, Wang L, Fang C, et al (2020) Clinical analysis of neonates born to mothers with 2019-nCoV pneumonia. Transl Pediatr 9(1):51-60 https://doi.org/10.21037/tp.2020.02.06

29. Breslin N, Baptiste C, Gyamfi-Bannerman C et al (2020) COVID19 infection among asymptomatic and symptomatic pregnant women: Two weeks of confirmed presentations to an affiliated pair of New York City hospitals. AJOG MFM 2(2):100118. https ://doi.org/10.1016/j.ajogmf.2020.100118

30. Dong L, Tian J, He S et al (2020) Possible vertical transmission of SARS-CoV-2 from an infected mother to her newborn. JAMA. https://doi.org/10.1001/jama.2020.4621

31. Zeng H, Xu C, Fan J et al (2020) Antibodies in infants born to mothers with COVID-19 pneumonia. JAMA 323(18):1848-1849. https://doi.org/10.1001/jama.2020.4861

32. Yudin MH, Steele DM, Sgro MD, Read SE, Kopplin P, Gough KA (2005) Severe acute respiratory syndrome in pregnancy. Obstet Gynecol 105:124-127. https://doi.org/10.1097/01.AOG.00001 51598.49129.de

33. Gasparyan AY, Misra DP, Yessirkepov M, Zimba O (2020) Perspectives of immune therapy in coronavirus disease 2019. J Korean Med Sci 35(18):e176. https://doi.org/10.3346/ jkms.2020.35.e176

34. Wu Z, McCoogan JM (2020) Characteristics of and important lessons from the coronavirus disease 2019 (CPVOD-19) outbreak in China: summary of a report of 72,314 cases from the Chinese Center for Disease Control and Prevention. JAMA 323(13):1239_ 1242. https://doi.org/10.1001/jama.2020.2648
35. Chen L, Li Q, Zheng D et al (2020) Clinical characteristics of pregnant women with COVID-19 in Wuhan. China N Engl J Med 382:e100. https://doi.org/10.1056/NEJMc2009226

36. Liu Y, Chen H, Tang K et al (2020) Clinical manifestations and outcome of SARS-CoV-2 infection during pregnancy. J Infect. https://doi.org/10.1016/j.jinf.2020.02.028

37. Luo Y, Yin K (2020) Management of pregnant women infected with COVID-19. Lancet Infect Dis. https://doi.org/10.1016/ S1473-3099(20)30191-2

38. ICNARC report on COVID-19 in critical care. In: ICNARC, ed., 2020. https://www.icnarc.org/Our-Audit/Audits/Cmp/Reports. Accessed 17Apr 2020

39. Liu D, Li L, Wu X et al (2020) Pregnancy and perinatal outcomes of women with coronavirus disease (COVID-19) Pneumonia: a preliminary analysis. Am J Roentgenol. https://doi. org/10.2214/AJR.20.23072

40. Wong SF, Chow KM, Leung TN et al (2004) Pregnancy and perinatal outcomes with the severe acute respiratory syndrome. Am J Obstet Gynecol 191:292-297. https://doi.org/10.1016/j. ajog.2003.11.019

41. Alfaraj SH, Al-Tawfiq JA, Memish ZA (2019) Middle east respiratory syndrome coronavirus (MERS-CoV) infection during pregnancy: report of two cases $\&$ review of the literature. J Microbiol Immunol Infect. 52:501-503. https://doi. org/10.1016/j.jmii.2018.04.005

42. Chen S, Huang B, Luo DJ et al (2020) Pregnant woman with new coronavirus infection: clinical characteristics and placental pathological analysis of three cases. Zhonghua Bing Li Xue Za Zhi 49:E005. https://doi.org/10.3760/cma.j.cn112151-20200 225-00138

43. Mullins E, Evans D, Viner R et al (2020) Coronavirus in pregnancy and delivery: a rapid review. Ultrasound in Obstet Gynaecol 55:586-592. https://doi.org/10.1002/uog.22014

44. Royal College of Obstetricians and Gynaecologists. Reducing the Risk of Venous Thromboembolism during Pregnancy and the Puerperium. Green-top Guideline No. 37a. Accessed 2015

45. Practical guidance for the prevention of thrombosis and management of coagulopathy and disseminated intravascular coagulation of patients infected with COVID-19 (2020) https://throm bosisuk.org/covid-19-thrombosis.php Accessed 07 Apr 2020

46. Reverter JC, Tàssies D, Font J, Monteagudo J, Escolar G, Ingelmo M, Ordinas A (1996) Hypercoagulable state in patients with the antiphospholipid syndrome is related to high induced tissue factor expression on monocytes and too low free protein s. Arterioscler Thromb Vasc Biol 16(11):1319-1326. https:// doi.org/10.1161/01.ATV.16.11.1319

47. Listing J, Gerhold K, Zink A (2012) The risk of infections associated with rheumatoid arthritis, with its comorbidity and treatment. Rheumatology (Oxford) 52:53-61. https://doi. org/10.1093/rheumatology/kes305

48. Tudesq J-J, Cartron G, Rivière S, Morquin D, Iordache L, Mahr A (2018) Clinical and microbiological characteristics of the infections in patients treated with rituximab for autoimmune and/or malignant hematological disorders. Autoimmun Rev 17:115-124. https://doi.org/10.1016/j.autrev.2017.11.015

49. EULAR guidance for patients during the Covid-19 outbreak. https://www.eular.org/eular_guidance_for_patients_covid19_ outbreak.cfm Accessed 19 Mar 2020

50. ACR guidance for patients during the Covid-19 outbreak. https ://www.rheumatology.org/announcements. Accessed 29 Mar 2020

51. BSR guidance for patients during the Covid-19 outbreak. https:// www.rheumatology.org.uk/News-Policy/Details/Covid19 Coronavirus-update-members Accessed 29 Mar 2020

52. Australian Rheumatology Association guidance for patients during the Covid-19 outbreak. https://arthritisaustralia.com.au/advic 
e-regarding-coronavirus-covid-19-from-the-australian-rheumatolo gy-association/ Accessed 19 Mar 2020

53. World Health Organization. (2004). AIDE-MEMOIRE. For infection prevention and control in a health care facility. https://apps. who.int/iris/handle/10665/130165 Accessed 2004.

54. World Health Organization. (2020). Clinical Management of Severe Acute Respiratory Infection When Novel Coronavirus (2019-nCoV) Infection is Suspected: Interim Guidance. Accessed 28 Jan 2020

55. Bin Chao. (2020) Chinese guidelines of diagnosis and treatment of COVID-19 (7th version). https://www.sforl.org/wp-content/uploa ds/2020/03/WUHAN-Experience.pdf Accessed 18 Mar 2020

56. Favalli E, Biggioggero M, Maioli G et al (2020) Baricitinib for COVID-19: a suitable treatment? Lancet Infect Dis. https://doi. org/10.1016/S1473-3099(20)30262-0

57. Cantini F, Niccoli L, Matarrese D et al (2020) Baricitinib therapy in COVID-19: A pilot study on the safety and clinical impact. J Infect. https://doi.org/10.1016/j.jinf.2020.04.017

58. Stebbing J, Phelan A, Griffin I et al (2020) COVID-19: combining antiviral and anti-inflammatory treatments. Lancet Infect Dis 20:400-402. https://doi.org/10.1016/S1473-3099(20)30132-8

59. Tisoncik JR, Korth MJ, Simmons CP, Farrar J, Martin TR, Katze MG (2012) Into the eye of the cytokine storm. Microbiol Mol Biology Rev Mmbr 76:16-32. https://doi.org/10.1128/ MMBR.05015-11

60. Shakoory B, Carcillo JA, Chatham WW, Amdur RL, Zhao H, Dinarello CA (2016) Interleukin-1 receptor blockade is associated with reduced mortality in sepsis patients with features of macrophage activation syndrome. Crit Care Med 44:275-281. https ://doi.org/10.1097/CCM.0000000000001402

61. Papagoras C, Voulgari PV, Drosos AA (2013) Atherosclerosis and cardiovascular disease in the spondyloarthritides, particularly ankylosing spondylitis and psoriatic arthritis. Clin Exp Rheumatol 31(4):612-620
62. Papagoras C, Markatseli TE, Saougou I et al (2014) Cardiovascular risk profile in patients with spondyloarthritis. Joint Bone Spine 81(1):57-63. https://doi.org/10.1016/j.jbspin.2013.03.019

63. National Psoriasis Foundation Medical Board COVID-19 Recommendations for patients with psoriatic disease. https://www.psori asis.org/advance/coronavirus Accessed 10 Apr 2020

64. Popescu C, Pintilie AM, Bojinca V, Balanescu A, Ionescu R (2014) Cardiovascular risk in psoriatic arthritis - a cross-sectional study. Maedica (Buchar) 9(1):19-24

65. Mathian A, Mahevas M, Rohmer J et al (2020) Clinical course of coronavirus disease 2019 (COVID-19) in a series of 17 patients with systemic lupus erythematosus under long-term treatment with hydroxychloroquine. Ann Rheum Dis. https://doi. org/10.1136/annrheumdis-2020-217566

66. Zhou F, Yu T, Du R et al (2020) Clinical course and risk factors for mortality of adult inpatients with COVID-19 in Wuhan, China: a retrospective cohort study. Lancet 395:1054-62. https:// doi.org/10.1016/S0140-6736(20)30566-3

67. ICNARC. Report on 2249 patients critically ill with COVID19. https://www.icnarc.org/DataServices/Attachments/Downl oad/76a7364b-4b76-ea11-9124-00505601089b Accessed 04 Apr 2020

68. Askanase AD, Khalili L, Buyon JP (2020) Thoughts on COVID19 and autoimmune diseases. Lupus Sci Med. https://doi. org/10.1136/lupus-2020-000396

Publisher's Note Springer Nature remains neutral with regard to jurisdictional claims in published maps and institutional affiliations. 